\title{
Characterization of African Rice Germplasm for Morphological and Yield Attributing Traits
}

\author{
V.G. Ishwarya Lakshmi ${ }^{1,4}$, C. Gireesh ${ }^{1 *}$, M.Sreedhar ${ }^{2}$, S. Vanisri ${ }^{3}$, P.S. Basavaraj ${ }^{1,4}$, B. \\ Muralidhara $^{1}$, M.S. Anantha ${ }^{1}$, G. Padmavathi ${ }^{1}$, A.R. Fiyaz ${ }^{1}$, B. Jyothi ${ }^{1}$, C. Suvarna \\ Rani $^{1}$, Bidyasagar Mandal ${ }^{1}$ and L.V. Subba Rao ${ }^{1}$ \\ ${ }^{1}$ ICAR-Indian Institute of Rice Research, Rajendranagar, Hyderabad - 500 030, India \\ ${ }^{2}$ MFPI-Quality control Lab, Department of Genetics and Plant Breeding, College of \\ Agriculture, Rajendranagar, Hyderabad \\ ${ }^{3}$ Department of Molecular Biology and Biotechnology, Institute of Biotechnology, \\ Rajendranagar, Hyderabad \\ ${ }^{4}$ Department of Genetics and Plant Breeding, College of Agriculture, PJTSAU, \\ Rajendranagar, Hyderabad
}

*Corresponding author

\section{A B S T R A C T}

\begin{tabular}{|c|}
\hline Keywords \\
\hline $\begin{array}{l}\text { Oryza glaberrima, } \\
\text { DUS, Genetic } \\
\text { variability, } \\
\text { Heritability, } \\
\text { Genetic advance }\end{array}$ \\
\hline Article Info \\
\hline $\begin{array}{l}\text { Accepted: } \\
12 \text { November } 2018 \\
\text { Available Online: } \\
10 \text { December } 2018\end{array}$ \\
\hline
\end{tabular}

\section{Ke y w o r d s}

Oryza glaberrima

DUS, Genetic

Heritability,

Genetic advance

Article Info

Accepted:

Available Online:

\section{Introduction}

Rice (Oryza sativa L.) is the principal food grain crop in India and it is being cultivated in 43.9 million ha with total of production of 109.6 million tonnes (Indiastat, 2016-17). Since the release of semi dwarf rice varieties in 1960's in India and elsewhere, the genetic
For the establishment of the distinctness among 31 accessions of Oryza glaberrima, eight morphological traits following Distinctness, Uniformity and Stability test (DUS) studied. Out of 31 accessions studied, two accessions viz., EC861804, EC861805 were found to be highly distinct as they possessed distinct traits namely purple split ligule, medium green leaves, erect flag leaf, presence of awn along with lodging tolerance indicating their usefulness as donors for crop improvement programmes. Analysis of variance revealed significant differences for all the characters studied. Phenotypic coefficient variation was higher than genotypic coefficient variation and magnitude of PCV and GCV was high for number of productive tillers and spikelets per panicle. High heritability coupled with high genetic advance was observed for days to 50 per cent flowering and number of spikelets per panicle which suggested the presence of high additive gene action which would respond to selection owing their high genetic variability and transmissibility. 
such as resistance to biotic, abiotic stresses (Fofana and Cloutier, 2008) along with early maturity. In addition, $O$. glaberrima is also a potential source of genes to enhance milling, cooking, eating qualities of indica rice. In India, O. glaberrima is used in limited extent for genetic improvement of indica rice as very less systematic efforts have been undertaken in India to utilize the wealth of African species for genetic improvement of indica rice varieties (Sarla and Swamy, 2005).

Characterization of a variety is useful to identify and avoid duplication, enabling rice breeders to exploit a wide range of genotypic diversities for further crop improvement practices to increase the rice productivity. Generally, the morphological traits are qualitative in nature and are stable over generations (Raut, 2003), making them more reliable as morphological markers for the characterization of varieties. Development of high yielding cultivars with wide adaptability is the ultimate aim of plant breeders. Knowledge of genetic variability present in a given crop species for the character under improvement is of paramount importance for the success of any plant breeding program for broadening the gene pool of crops (Ahmad et al., 2011). Heritability provides information on the extent to which a particular morphogenetic character can be transmitted to successive generations and also influences the choice of selection procedures used by the plant breeder to decide which selection methods would be most useful to improve the character (waqar et al., 2008). Characters with high heritability can easily be fixed with simple selection, resulting in quick progress. However, it has been accentuated that heritability alone has no practical importance without genetic advance (Najeeb et al., 2009). A systematic study analysis of genetic diversity is essential to exploit the inherent variability and to broaden the genetic base of rice cultivars (Rout et al., 2017). Thus, the present study was conducted to characterize understand the performance of African rice germplasm for eight morphological traits along with a study on genetic variability, heritability and genetic advance for grain yield and its component characters. The knowledge gained in the present study is will be useful for exploitation of genetic wealth of African rice for genetic improvement of Indian cultivars.

\section{Materials and Methods}

Thirty one $O$. glaberrima lines received from IRRI (International Rice Research Institute) were sown in dry bed during Kharif2017 at the ICAR-Indian Institute of Rice Research, Hyderabad. Twenty one days old seedlings of each accession were transplanted by adopting a spacing of $20 \mathrm{~cm}$ between rows and $15 \mathrm{~cm}$ between plants in a Randomized Block Design with two replications. Morphological characterization was carried out using eight DUS characters. Visual observations were recorded on single plant basis on five randomly selected plants in each accession at appropriate growth stages on eight qualitative characters viz., Coleoptile colour, leaf intensity of green colour, culm type, leaf anthocyanin colouration, leaf ligule, flag leaf characteristics, panicle awn and stem anthocyanin colouration. Data was recorded on 10 quantitative characters namely, days to $50 \%$ flowering, plant height $(\mathrm{cm})$, panicle length $(\mathrm{cm})$, number of productive tillers, number of spikelets per panicle, 1000seed weight (g) and grain yield per plant (g), kernel length $(\mathrm{mm})$, kernel breadth $(\mathrm{mm})$ and $\mathrm{L} / \mathrm{B}$ ratio. Replicated mean data of each character was subjected to analysis of variance following Panse and Sukatme (1985). Genotypic, phenotypic and environmental variance along with heritability and genetic advance were estimated for all the characters. The phenotypic (PCV) and genotypic (GCV) coefficients of variation, heritability (broad sense) and genetic advance were estimated by 
the formulae suggested by Burton et al., (1952) and Johnson et al., (1955).

\section{Results and Discussion}

Among the eight qualitative characters studied in morphological characterization of the accessions (Table 1), three characters (Table 2) viz., absence of coleoptile colour, leaf anthocyanin colouration and presence of leaf ligule were common in all the 31 accessions of O. glaberrima. Remaining five characters were unique and distinct among the accessions. The coleoptile was colourless in all the accessions of $O$. glaberrima. Regarding leaf characteristics, intensity of green colour in leaves was light in 18 accessions $(58.06 \%)$ and medium green in the remaining 13 accessions. The anthocyanin colouration of leaves was absent in all the accessions of $O$. glaberrima, while all the 31 accessions of $O$. glaberrima had the leaf ligule with split shape in two accessions (EC 861804 and 861814 and acute in the remaining 29 accessions. The colour of ligule was also distinct across the accessions as it was white in 26 and purple in five accessions (EC861791, EC861796, EC861805, EC861819 and EC861817). With respect to the culm type, out of the 31 accessions of $O$. glaberrima, 27 accessions (87.09\%) showed erect culm, one accession (EC861791) had semi erect culm, while spreading culm was observed in three accessions (EC861807, EC861812 and EC861813). The $O$. glaberrima accessions having erect type of culm can show lodging resistance. Regarding the attitude of the flag leaf blade, it was erect in six accessions (EC 861799, EC861804, EC861807, EC861810, EC861814 and EC861816), semi-erect in 19 and horizontal in the remaining six accessions (EC861792, EC816794, EC861797, EC861784, EC861811 and EC861815). The six accessions of $O$. glaberrima having erect flag leaf can serve as donor lines for improvement in rice breeding programmes.
For the panicle awn, nine accessions (EC861795, EC861799, EC861803, EC861812, EC861813, EC861814, EC861815, EC861817 and EC861818) recorded the presence of awn while it was absent in the remaining 22 accessions. With regard to the stem anthocyanin colouration, 13 accessions (41.93\%) recorded the purple colouration while 18 accessions did not show anthocyanin colouration on stem. Two accessions viz., EC861804, EC861805 were found to be highly distinct as they possessed distinct traits namely purple split ligule, medium green leaves, erect flag leaf, presence of awn along with lodging tolerance indicating their usefulness as donors for crop improvement programmes (Table 3).

Analysis of variance (Table 4) revealed significant differences among the accessions under study for all the ten traits indicating the presence of considerable genetic variability in the experimental material. A study of genetic parameters (Table 5) revealed that phenotypic and genotypic coefficients of variation were high for number of productive tillers per plant and spikelets per panicle The values of GCV and PCV were moderate for days to 50 per cent flowering, plant height, productive tillers, 1000 seed weight, grain yield per plant and kernel length while low for panicle length, kernel breadth and L/B ratio. These results are in accordance with findings of Rusdiansyah et al., (2017) for high GCV and PCV, Abebe et al., (2017) for moderate GCV, PCV and Edukondalu et al., (2017) for low GCV and $\mathrm{PCV}$ in rice (O. sativa L.).High heritability coupled with high genetic advance was observed for days to 50 per cent flowering, plant height and number of spikelets per panicle indicated the preponderance of high additive type of gene action in the inheritance of these characters which can be further improved by following simple selection procedure as suggested by Abebe et al., (2017) (Fig. 1 and Table 6). 
Table.1 List of Oryza glaberrima accessions used in the present study

\begin{tabular}{|c|c|c|c|}
\hline S.No & Accession No. & Origin & Biological status of accession \\
\hline 1 & EC861784 & Guinea & Traditional cultivar/ Landrace \\
\hline 2 & EC861785 & Guinea & Traditional cultivar/ Landrace \\
\hline 3 & EC861786 & Guinea & Traditional cultivar/ Landrace \\
\hline 4 & EC861787 & Guinea & Traditional cultivar/ Landrace \\
\hline 5 & EC861790 & Guinea & Traditional cultivar/ Landrace \\
\hline 6 & EC861791 & Guinea & Traditional cultivar/ Landrace \\
\hline 7 & EC861792 & Guinea & Traditional cultivar/ Landrace \\
\hline 8 & EC861794 & Guinea & Traditional cultivar/ Landrace \\
\hline 9 & EC861795 & Guinea & Traditional cultivar/ Landrace \\
\hline 10 & EC861796 & Guinea & Traditional cultivar/ Landrace \\
\hline 11 & EC861797 & Guinea & Traditional cultivar/ Landrace \\
\hline 12 & EC861799 & Guinea & Traditional cultivar/ Landrace \\
\hline 13 & EC861801 & Guinea & Traditional cultivar/ Landrace \\
\hline 14 & EC861802 & Guinea & Traditional cultivar/ Landrace \\
\hline 15 & EC861803 & Guinea & Traditional cultivar/ Landrace \\
\hline 16 & EC861804 & Guinea & Traditional cultivar/ Landrace \\
\hline 17 & EC861805 & Guinea & Traditional cultivar/ Landrace \\
\hline 18 & EC861807 & Guinea & Traditional cultivar/ Landrace \\
\hline 19 & EC861808 & Guinea & Traditional cultivar/ Landrace \\
\hline 20 & EC861809 & Guinea & Traditional cultivar/ Landrace \\
\hline 21 & EC861810 & Guinea & Traditional cultivar/ Landrace \\
\hline 22 & EC861811 & Guinea & Traditional cultivar/ Landrace \\
\hline 23 & EC861812 & Guinea & Traditional cultivar/ Landrace \\
\hline 24 & EC861813 & Guinea & Traditional cultivar/ Landrace \\
\hline 25 & EC861814 & Guinea & Traditional cultivar/ Landrace \\
\hline 26 & EC861815 & Guinea & Traditional cultivar/ Landrace \\
\hline 27 & EC861816 & Guinea & Traditional cultivar/ Landrace \\
\hline 28 & EC861817 & Guinea & Traditional cultivar/ Landrace \\
\hline 29 & EC861818 & Guinea & Traditional cultivar/ Landrace \\
\hline 30 & EC861819 & Malaysia & Wild \\
\hline 31 & EC861820 & Malaysia & Wild \\
\hline
\end{tabular}


Table.2 Characterization of 31 accessions of O. glaberrima for morphological traits

\begin{tabular}{|c|c|c|c|c|c|c|c|c|c|}
\hline S.NO. & Accession & $\begin{array}{c}\text { Coleoptile } \\
\text { colour }\end{array}$ & $\begin{array}{c}\text { Intensity of } \\
\text { green colour } \\
\text { in leaves }\end{array}$ & $\begin{array}{c}\text { Leaf } \\
\text { anthocyanin } \\
\text { colouration }\end{array}$ & $\begin{array}{c}\text { Leaf } \\
\text { ligule }\end{array}$ & Culm type & $\begin{array}{l}\text { Flag leaf } \\
\text { attitude }\end{array}$ & $\begin{array}{c}\text { Panicle } \\
\text { awn }\end{array}$ & $\begin{array}{c}\text { Stem } \\
\text { anthocyanin } \\
\text { colouration }\end{array}$ \\
\hline 1 & EC 861784 & colourless & light & absent & present & erect & horizontal & absent & absent \\
\hline 2 & EC 861785 & colourless & light & absent & present & erect & semi erect & absent & absent \\
\hline 3 & EC 861786 & colourless & light & absent & present & erect & semi erect & absent & absent \\
\hline 4 & EC 861787 & colourless & medium & absent & present & erect & semi erect & absent & absent \\
\hline 5 & EC 861790 & colourless & medium & absent & present & erect & semi erect & absent & absent \\
\hline 6 & EC 861791 & colourless & medium & absent & present & semi erect & semi erect & absent & absent \\
\hline 7 & EC 861792 & colourless & light & absent & present & erect & horizontal & absent & absent \\
\hline 8 & EC 861794 & colourless & medium & absent & present & erect & horizontal & absent & present \\
\hline 9 & EC 861795 & colourless & light & absent & present & erect & semi erect & present & present \\
\hline 10 & EC 861796 & colourless & light & absent & present & erect & semi erect & absent & absent \\
\hline 11 & EC 861797 & colourless & light & absent & present & erect & horizontal & absent & absent \\
\hline 12 & EC 861799 & colourless & light & absent & present & erect & erect & present & present \\
\hline 13 & EC 861801 & colourless & medium & absent & present & erect & semi erect & absent & present \\
\hline 14 & EC 861802 & colourless & light & absent & present & erect & semi erect & absent & present \\
\hline 15 & EC 861803 & colourless & light & absent & present & erect & semi erect & present & present \\
\hline 16 & EC 861804 & colourless & medium & absent & present & erect & erect & absent & absent \\
\hline 17 & EC 861805 & colourless & medium & absent & present & erect & semi erect & absent & present \\
\hline 18 & EC 861807 & colourless & medium & absent & present & spreading & semi erect & absent & present \\
\hline 19 & EC 861808 & colourless & medium & absent & present & erect & semi erect & absent & absent \\
\hline 20 & EC 861809 & colourless & medium & absent & present & erect & semi erect & absent & present \\
\hline 21 & EC 861810 & colourless & light & absent & present & erect & erect & absent & absent \\
\hline 22 & EC 861811 & colourless & light & absent & present & erect & horizontal & absent & absent \\
\hline 23 & EC 861812 & colourless & medium & absent & present & spreading & semi erect & present & absent \\
\hline 24 & EC 861813 & colourless & light & absent & present & spreading & semi erect & present & present \\
\hline 25 & EC 861814 & colourless & light & absent & present & erect & erect & present & present \\
\hline 26 & EC 861815 & colourless & light & absent & present & erect & horizontal & present & absent \\
\hline 27 & EC 861816 & colourless & medium & absent & present & erect & erect & absent & absent \\
\hline 28 & EC 861817 & colourless & medium & absent & present & erect & semi erect & present & absent \\
\hline 29 & EC 861818 & colourless & light & absent & present & erect & semi erect & present & present \\
\hline 30 & EC 861819 & colourless & light & absent & present & erect & semi erect & absent & present \\
\hline 31 & EC 861820 & colourless & light & absent & present & erect & semi erect & absent & absent \\
\hline
\end{tabular}


Table.3 Frequency distribution of morphological traits in 31 accessions of $O$. glaberrima

\begin{tabular}{|c|c|c|c|c|c|}
\hline S.No. & Character & Status & $\begin{array}{c}\text { No. of } \\
\text { accessions }\end{array}$ & Frequency & Accessions \\
\hline 1 & $\begin{array}{l}\text { Coleoptile } \\
\text { colour }\end{array}$ & Colourless & 31 & $100 \%$ & $\begin{array}{l}\text { EC 861784, 861785, 861786, 861787, 861790, } \\
861791,861792,861794,861795,861796, \\
861797,861799,861801,861802,861803, \\
861804,861805,861807,861808,861809, \\
861810,861811,861812,861813,861814, \\
861815,861816,861817,861818,861819,861820\end{array}$ \\
\hline \multirow[t]{2}{*}{2} & \multirow{2}{*}{$\begin{array}{l}\text { Intensity of } \\
\text { green colour in } \\
\text { leaves }\end{array}$} & Light & 18 & $58.06 \%$ & $\begin{array}{l}\text { EC 861784, 861785, 861786, 861792, 861795, } \\
861796,861797,861799,861802,861803,861810, \\
861811,861813,861814,861815,861818,861819, \\
861820\end{array}$ \\
\hline & & Medium & 13 & $41.93 \%$ & $\begin{array}{l}\text { EC } 861787,861790,861791,861794,861801, \\
861804,861805,861807,861808,861809,861812, \\
861816,861817\end{array}$ \\
\hline \multirow[t]{2}{*}{3} & \multirow{2}{*}{$\begin{array}{l}\text { Leaf } \\
\text { anthocyanin } \\
\text { colouration }\end{array}$} & Present & 0 & $0 \%$ & - \\
\hline & & Absent & 31 & $100 \%$ & $\begin{array}{l}\text { EC 861784, 861785, 861786, 861787, 861790, } \\
861791,861792,861794,861795,861796, \\
861797,861799,861801,861802,861803, \\
861804,861805,861807,861808,861809 \\
861810,861811,861812,861813,861814, \\
861815,861816,861817,861818,861819 \\
861820\end{array}$ \\
\hline
\end{tabular}




\begin{tabular}{|c|c|c|c|c|c|}
\hline S.No. & Character & Status & $\begin{array}{c}\text { No. of } \\
\text { accessions }\end{array}$ & Frequency & Accessions \\
\hline \multirow[t]{2}{*}{4} & \multirow[t]{2}{*}{ Leaf ligule } & Present & 31 & $100 \%$ & $\begin{array}{l}\text { EC 861784, 861785, 861786, 861787, 861790, 861791, } \\
861792,861794,861795,861796,861797,861799,861801, \\
861802,861803,861804,861805,861807,861808,861809, \\
861810,861811,861812,861813,861814,861815,861816, \\
861817,861818,861819,861820\end{array}$ \\
\hline & & Absent & 0 & $0 \%$ & - \\
\hline \multirow[t]{3}{*}{5} & \multirow[t]{3}{*}{ Culm type } & Erect & 27 & $87.09 \%$ & $\begin{array}{l}\text { EC 861784, 861785, 861786, 861787, 861790, 861792, } \\
861795,861796,861797,861799,861801,861802,861803, \\
861804,861805,861808,861810,861811,861814,861815, \\
861816,861817,861818,861819,861820\end{array}$ \\
\hline & & Semi erect & 1 & $0.03 \%$ & EC 861791 \\
\hline & & Spreading & 3 & $0.09 \%$ & EC $861807,861812,861813$ \\
\hline \multirow[t]{3}{*}{6} & \multirow{3}{*}{$\begin{array}{l}\text { Flag leaf } \\
\text { attitude }\end{array}$} & Erect & 6 & $19.35 \%$ & EC $861799,861804,861807,861810,861814,861816$ \\
\hline & & Semi erect & 19 & $61.29 \%$ & $\begin{array}{l}\text { EC 861785,861786, 861787,861790, 861791,861795, } \\
861796,861801,861802,861803,861805,861808,\end{array}$ \\
\hline & & Horizontal & 6 & $19.35 \%$ & EC $861784,86179,861794,86179,861811,861815$ \\
\hline \multirow[t]{2}{*}{7} & \multirow[t]{2}{*}{ Panicle awn } & Present & 9 & $29.03 \%$ & $\begin{array}{l}\text { EC } 861795,861799,861803,861812,861813,861814, \\
861815,861817,861818\end{array}$ \\
\hline & & Absent & 22 & $70.96 \%$ & $\begin{array}{l}\text { EC } 861784,861785,861786,861787,861790,861791, \\
861792,861794,861796,861797,861801,861802,861804, \\
861805,861807,861808,861809,861811,861810,861816, \\
861819,861820\end{array}$ \\
\hline \multirow[t]{2}{*}{8} & \multirow{2}{*}{$\begin{array}{l}\text { Stem } \\
\text { anthocyanin } \\
\text { colouration }\end{array}$} & Present & 13 & $41.93 \%$ & $\begin{array}{l}\text { EC 861794, 861795, 861799, 861801, 861802, 861803, } \\
861805,861807,861809,861813,861814,861818,861819\end{array}$ \\
\hline & & Absent & 18 & $58.06 \%$ & $\begin{array}{l}\text { EC } 861784,861785,861786,861787,861790,861791, \\
861792,861796,861797,861804,861808,861810,861811, \\
861812,861815,861816,861817,861820\end{array}$ \\
\hline
\end{tabular}


Table.4 Analysis of variance for yield and yield attributing traits in rice Oryza glaberrima accessions

\begin{tabular}{|c|c|c|c|c|}
\hline $\begin{array}{l}\text { S. } \\
\text { No. }\end{array}$ & Character & $\begin{array}{l}\text { Replication } \\
\quad(\text { d.f. }=1)\end{array}$ & $\begin{array}{c}\text { Treatment } \\
\text { (d.f.=30) }\end{array}$ & $\begin{array}{c}\text { Error } \\
\text { (d.f. }=30)\end{array}$ \\
\hline 1 & Days to $50 \%$ flowering & 41.30 & $283.00 * *$ & 12.58 \\
\hline 2 & Plant height $(\mathrm{cm})$ & 0.16 & $476.159 * *$ & 57.39 \\
\hline 3 & Number of productive tillers & 1.19 & $36.29 * *$ & 2.21 \\
\hline 4 & Panicle length $(\mathrm{cm})$ & 3.71 & $8.64 * *$ & 3.63 \\
\hline 5 & No. of spikelets per panicle & 71.85 & $2257.44 * *$ & 279.08 \\
\hline 6 & 1000 grain weight (g) & 17.80 & $16.12^{* *}$ & 5.06 \\
\hline 7 & Grain yield per plant (g) & 1.45 & $3.54 * *$ & 0.36 \\
\hline 8 & Kernel length (mm) & 0.11 & $0.48 * *$ & 0.18 \\
\hline 9 & Kernel breadth (mm) & 0.03 & $0.09 * *$ & 0.02 \\
\hline 10 & $\mathrm{~L} / \mathrm{B}$ ratio & 0.01 & $0.13 * *$ & 0.05 \\
\hline \multicolumn{5}{|c|}{ Significant at $1 \%$ level $*$ Significant at $5 \%$ level } \\
\hline
\end{tabular}

Table.5 Magnitude of variability, heritability and genetic advance for yield and yield attributing traits in Oryza glaberrima accessions

\begin{tabular}{|l|r|r|r|r|}
\hline \multicolumn{1}{|c|}{ Characters } & $\begin{array}{c}\text { PCV } \\
(\%)\end{array}$ & \multicolumn{1}{c|}{$\begin{array}{c}\text { GCV } \\
(\%)\end{array}$} & $\begin{array}{c}\text { Heritability in } \\
\text { broad } \\
\text { sense }\left(\mathrm{h}^{2}\right)(\%)\end{array}$ & $\begin{array}{r}\text { Genetic } \\
\text { Advance } \\
(\text { at 5\%) }\end{array}$ \\
\hline Days to 50\% flowering & 10.38 & 9.93 & 91.49 & 22.91 \\
\hline Plant height (cm) & 12.32 & 10.92 & 78.49 & 26.41 \\
\hline No. of productive tillers per plant & 34.38 & 32.35 & 88.51 & 8.00 \\
\hline Panicle length (cm) & 9.33 & 6.35 & 40.84 & 2.08 \\
\hline No. of spikelets per panicle & 29.98 & 26.48 & 77.99 & 57.22 \\
\hline 1000 grain weight (g) & 18.45 & 13.33 & 52.19 & 3.4 \\
\hline Grain yield per plant (g) & 16.58 & 14.98 & 81.63 & 2.35 \\
\hline Kernel length (mm) & 16.36 & 15.33 & 87.86 & 2.51 \\
\hline Kernel breadth (mm) & 8.55 & 6.13 & 51.54 & 0.26 \\
\hline L/B ratio & 10.34 & 7.00 & 45.86 & 0.28 \\
\hline
\end{tabular}


Int.J.Curr.Microbiol.App.Sci (2018) 7(12): 1288-1303

Table.6 Mean performance of O.glaberrima accessions for yield and yield attributing traits

\begin{tabular}{|c|c|c|c|c|c|c|c|c|c|c|c|}
\hline S.NO. & Accession & $\begin{array}{c}\text { Days to } \\
50 \% \\
\text { flowering }\end{array}$ & $\begin{array}{l}\text { Plant } \\
\text { height } \\
(\mathrm{cm})\end{array}$ & $\begin{array}{l}\text { Productive } \\
\text { tillers }\end{array}$ & $\begin{array}{c}\text { Panicle } \\
\text { length } \\
(\mathrm{cm})\end{array}$ & $\begin{array}{c}\text { Spikelets/ } \\
\text { Panicle }\end{array}$ & $\begin{array}{c}1000 \\
\text { seed } \\
\text { weight } \\
(\mathrm{g})\end{array}$ & $\begin{array}{l}\text { Yield/ } \\
\text { Plant } \\
(\mathrm{g})\end{array}$ & $\begin{array}{c}\text { Kernel } \\
\text { length } \\
(\mathrm{mm})\end{array}$ & $\begin{array}{c}\text { Kernel } \\
\text { breadth } \\
(\mathrm{mm})\end{array}$ & $\begin{array}{l}\mathrm{L} / \mathrm{B} \\
\text { ratio }\end{array}$ \\
\hline 1 & EC 861784 & 126.0 & 127.0 & 12.0 & 26.7 & 110.0 & 15.9 & 8.2 & 7.2 & 2.6 & 2.7 \\
\hline 2 & EC 861785 & 125.0 & 150.9 & 14.0 & 29.1 & 102.0 & 14.8 & 7.2 & 8.0 & 3.4 & 2.3 \\
\hline 3 & EC 861786 & 139.0 & 138.8 & 14.0 & 24.2 & 110.0 & 13.7 & 8.1 & 7.8 & 2.7 & 2.8 \\
\hline 4 & EC 861787 & 120.0 & 128.5 & 8.0 & 24.0 & 159.0 & 15.5 & 7.2 & 7.3 & 3.0 & 2.4 \\
\hline 5 & EC 861790 & 137.0 & 122.2 & 14.0 & 27.0 & 119.0 & 16.3 & 9.7 & 8.3 & 3.0 & 2.7 \\
\hline 6 & EC 861791 & 146.0 & 122.8 & 13.0 & 26.0 & 87.0 & 11.3 & 6.5 & 7.5 & 2.7 & 2.7 \\
\hline 7 & EC 861792 & 126.0 & 132.9 & 14.0 & 23.4 & 107.0 & 14.6 & 7.1 & 8.7 & 2.8 & 3.0 \\
\hline 8 & EC 861794 & 116.0 & 133.3 & 11.0 & 28.2 & 145.0 & 17.6 & 8.5 & 7.9 & 2.7 & 2.8 \\
\hline 9 & EC 861795 & 119.0 & 144.6 & 9.0 & 23.3 & 120.0 & 18.7 & 9.7 & 9.0 & 3.0 & 3.0 \\
\hline 10 & EC 861796 & 113.0 & 144.2 & 10.0 & 27.5 & 121.0 & 22.0 & 7.3 & 8.4 & 2.8 & 2.9 \\
\hline 11 & EC 861797 & 123.0 & 122.7 & 8.0 & 25.4 & 113.0 & 17.6 & 9.0 & 7.4 & 2.9 & 2.5 \\
\hline 12 & EC 861799 & 117.0 & 132.7 & 15.0 & 27.1 & 94.0 & 19.2 & 9.1 & 8.3 & 2.9 & 2.8 \\
\hline 13 & EC 861801 & 121.0 & 136.9 & 7.0 & 27.3 & 98.0 & 16.2 & 9.1 & 8.2 & 3.1 & 2.6 \\
\hline 14 & EC 861802 & 120.0 & 133.8 & 10.0 & 27.7 & 108.0 & 19.3 & 9.4 & 8.3 & 2.9 & 2.8 \\
\hline 15 & EC 861803 & 114.0 & 140.6 & 13.0 & 23.8 & 84.0 & 15.8 & 8.3 & 8.2 & 2.7 & 3.0 \\
\hline 16 & EC 861804 & 100.0 & 138.8 & 16.0 & 23.0 & 87.0 & 22.8 & 8.2 & 8.3 & 3.2 & 2.5 \\
\hline 17 & EC 861805 & 140.0 & 140.1 & 9.0 & 24.4 & 80.0 & 12.1 & 6.5 & 8.3 & 3.2 & 2.5 \\
\hline 18 & EC 861807 & 112.0 & 144.6 & 15.0 & 24.3 & 113.0 & 18.2 & 8.2 & 8.1 & 3.2 & 2.5 \\
\hline 19 & EC 861808 & 114.0 & 130.7 & 14.0 & 23.3 & 118.0 & 18.4 & 7.9 & 8.5 & 3.0 & 2.8 \\
\hline 20 & EC 861809 & 104.0 & 136.5 & 12.0 & 29.3 & 101.0 & 18.1 & 10.8 & 8.4 & 2.9 & 2.9 \\
\hline 21 & EC 861810 & 116.0 & 118.0 & 12.0 & 24.2 & 138.0 & 19.2 & 8.9 & 8.7 & 3.0 & 2.9 \\
\hline 22 & EC 861811 & 112.0 & 139.3 & 8.0 & 24.7 & 110.0 & 19.0 & 10.1 & 8.5 & 2.9 & 2.9 \\
\hline 23 & EC 861812 & 111.0 & 124.3 & 10.0 & 22.6 & 110.0 & 22.1 & 7.1 & 8.0 & 3.0 & 2.6 \\
\hline 24 & EC 861813 & 131.0 & 128.2 & 15.0 & 22.8 & 110.0 & 16.1 & 12.8 & 8.8 & 2.4 & 3.6 \\
\hline 25 & EC 861814 & 109.0 & 138.2 & 7.0 & 24.4 & 102.0 & 20.7 & 7.9 & 8.5 & 2.8 & 2.9 \\
\hline 26 & EC 861815 & 104.0 & 150.8 & 14.0 & 23.9 & 115.0 & 15.8 & 7.8 & 9.0 & 2.8 & 3.2 \\
\hline 27 & EC 861816 & 86.0 & 117.3 & 7.0 & 23.0 & 117.0 & 19.8 & 7.8 & 8.5 & 2.8 & 3.0 \\
\hline 28 & EC 861817 & 106.0 & 147.5 & 11.0 & 25.7 & 104.0 & 19.3 & 8.2 & 8.1 & 2.7 & 2.9 \\
\hline 29 & EC 861818 & 109.0 & 155.2 & 7.0 & 23.6 & 126.0 & 17.9 & 8.3 & 8.2 & 2.8 & 2.8 \\
\hline 30 & EC 861819 & 103.0 & 147.2 & 8.0 & 22.5 & 94.0 & 20.0 & 8.0 & 8.7 & 2.9 & 3.0 \\
\hline 31 & EC 861820 & 118.0 & 152.0 & 13.0 & 27.2 & 91.0 & 19.8 & 6.8 & 9.2 & 2.9 & 3.1 \\
\hline
\end{tabular}


Fig.1 Bar graph for morphological characterization of 31 accessions of $O$. glaberrima
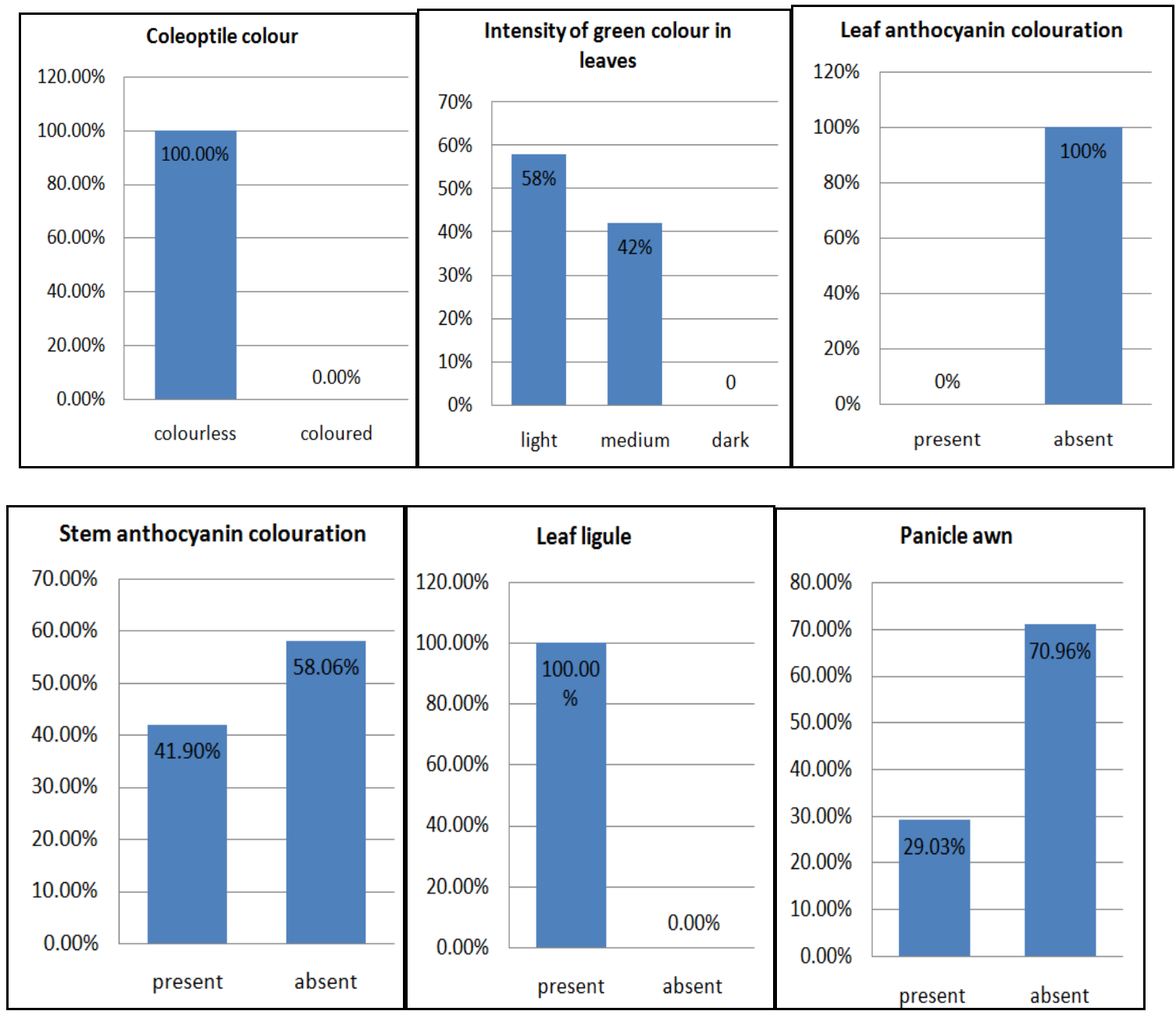

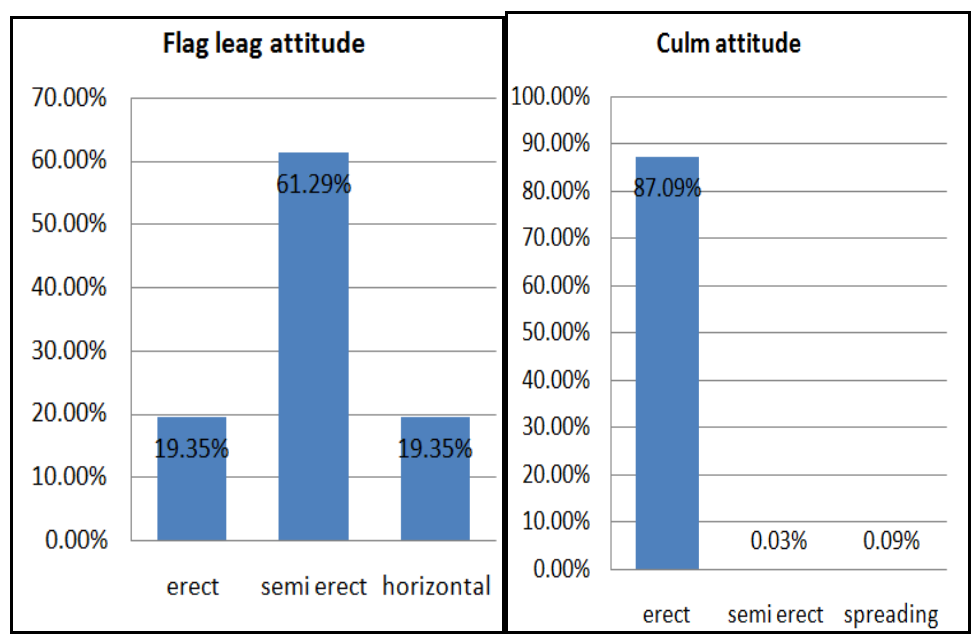


Plate.3 Variation in ligule colour and shape

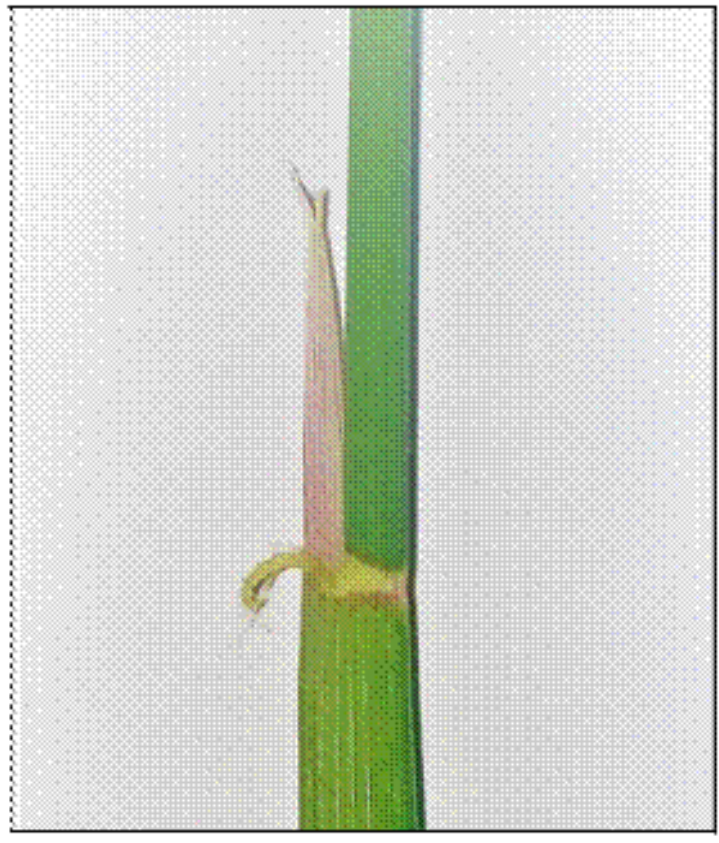

Purple Eigule

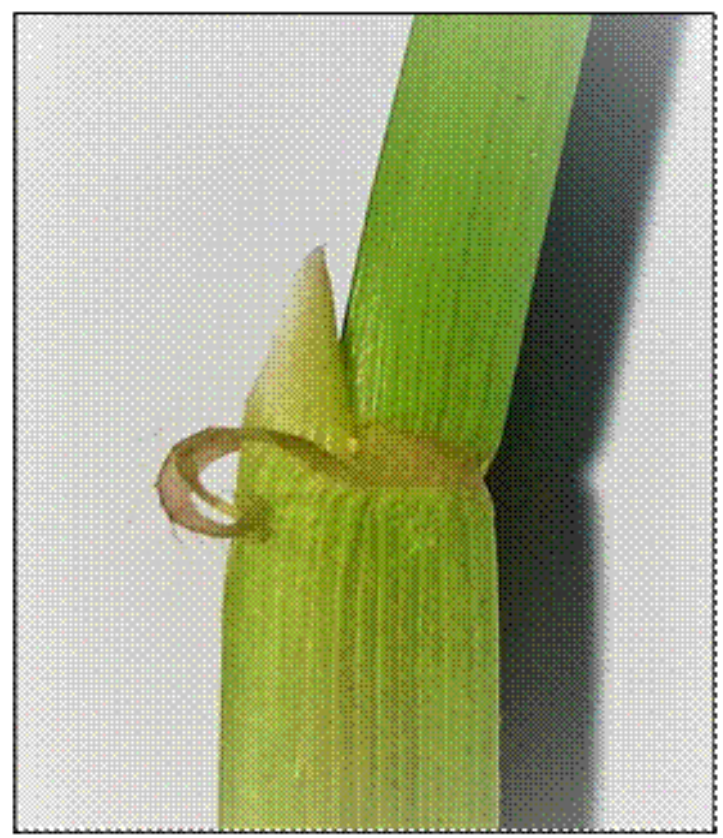

Acute Ligule

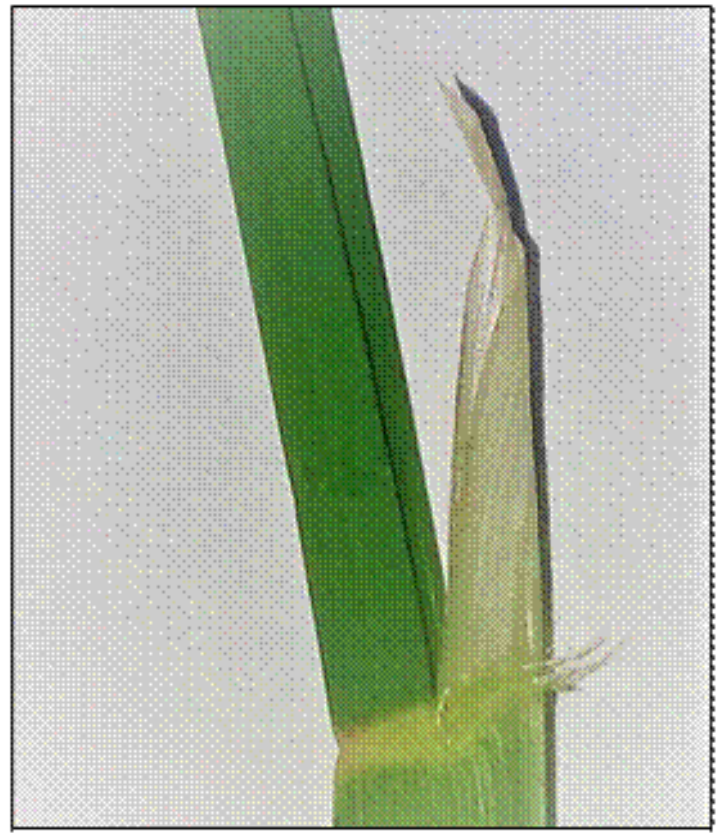

Colourless Ligule

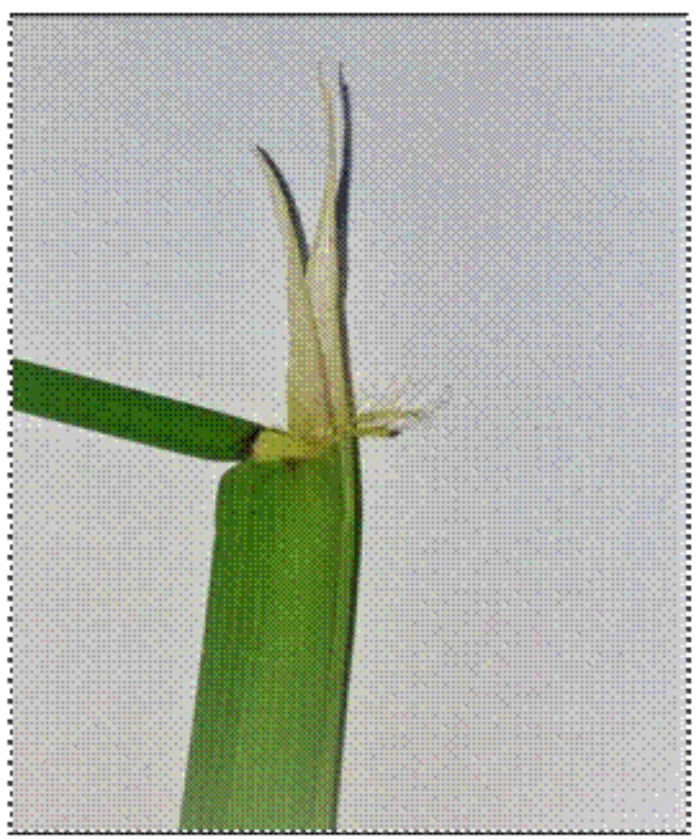

Split Ligule 
Plate.4 Variation in culm attitude

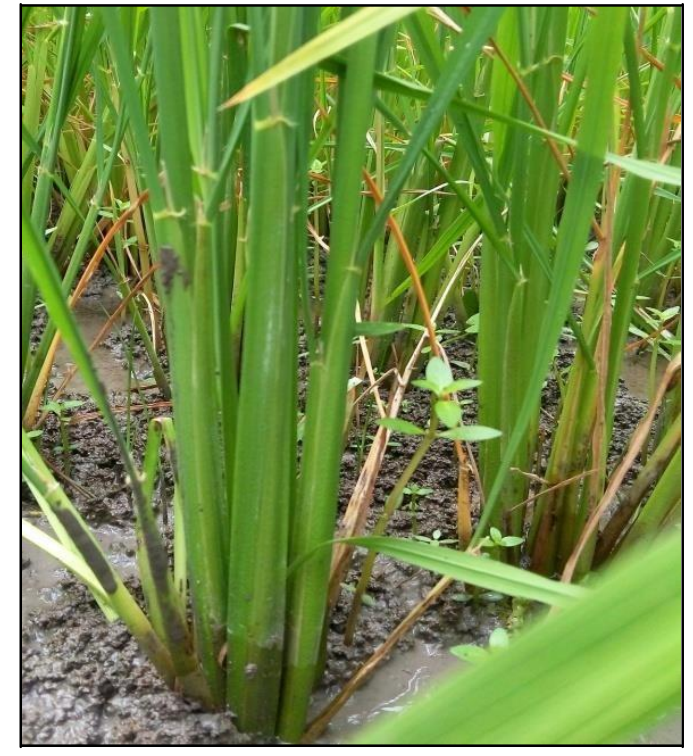

Erect

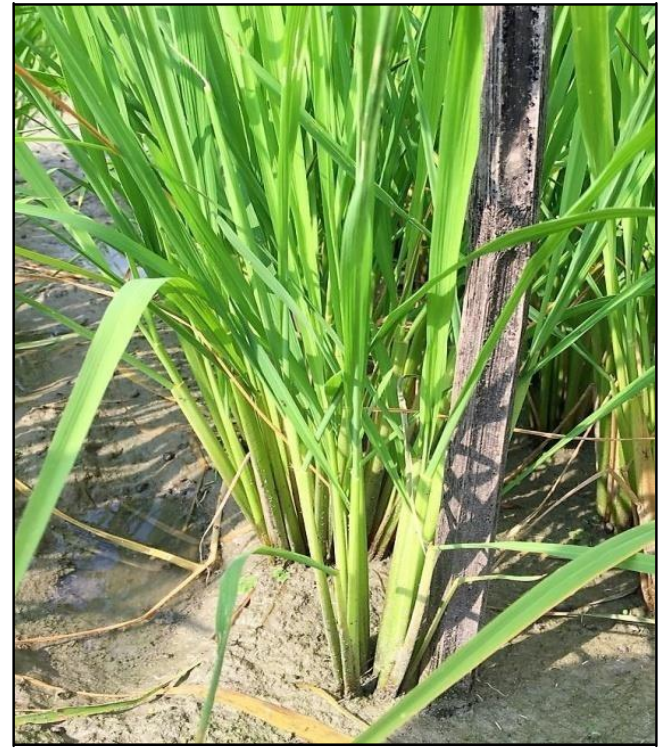

Semi Erect

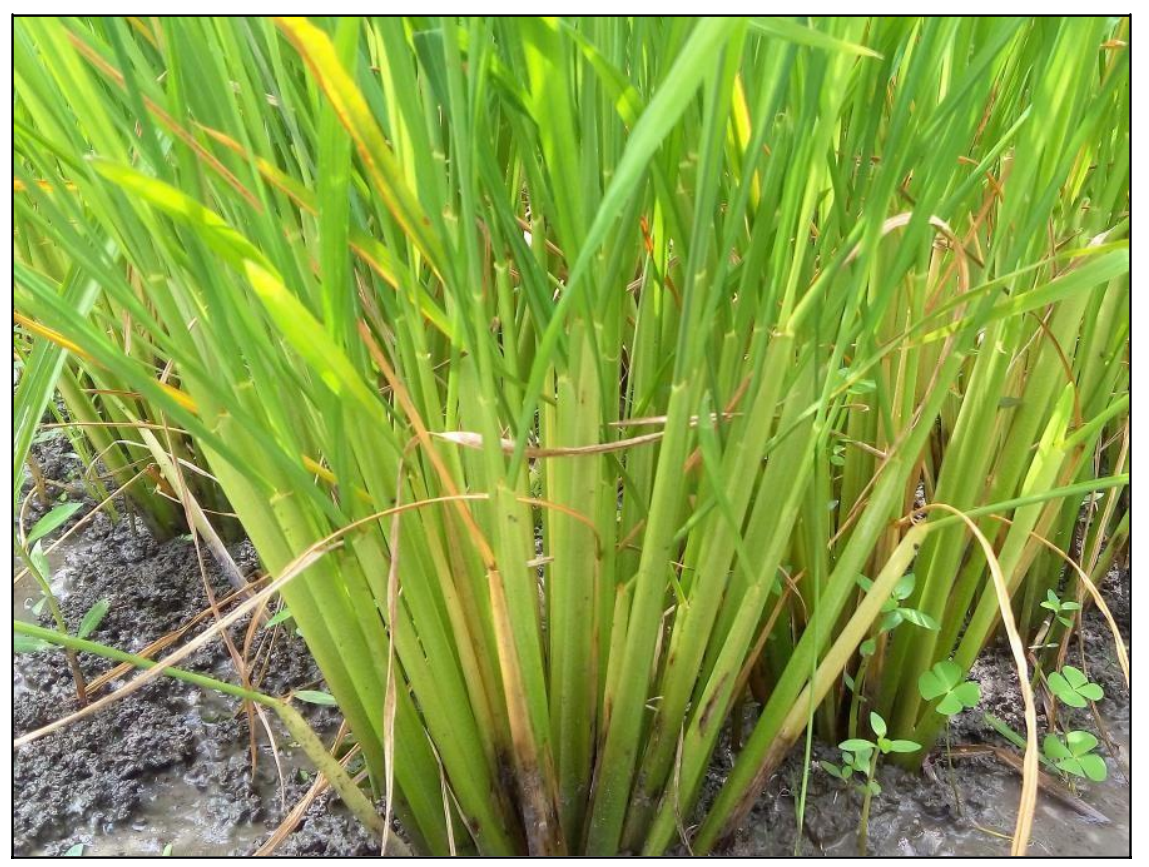

Spreading 
Int.J.Curr.Microbiol.App.Sci (2018) 7(12): 1288-1303

Plate.5 Variation in attitude of flag leaf

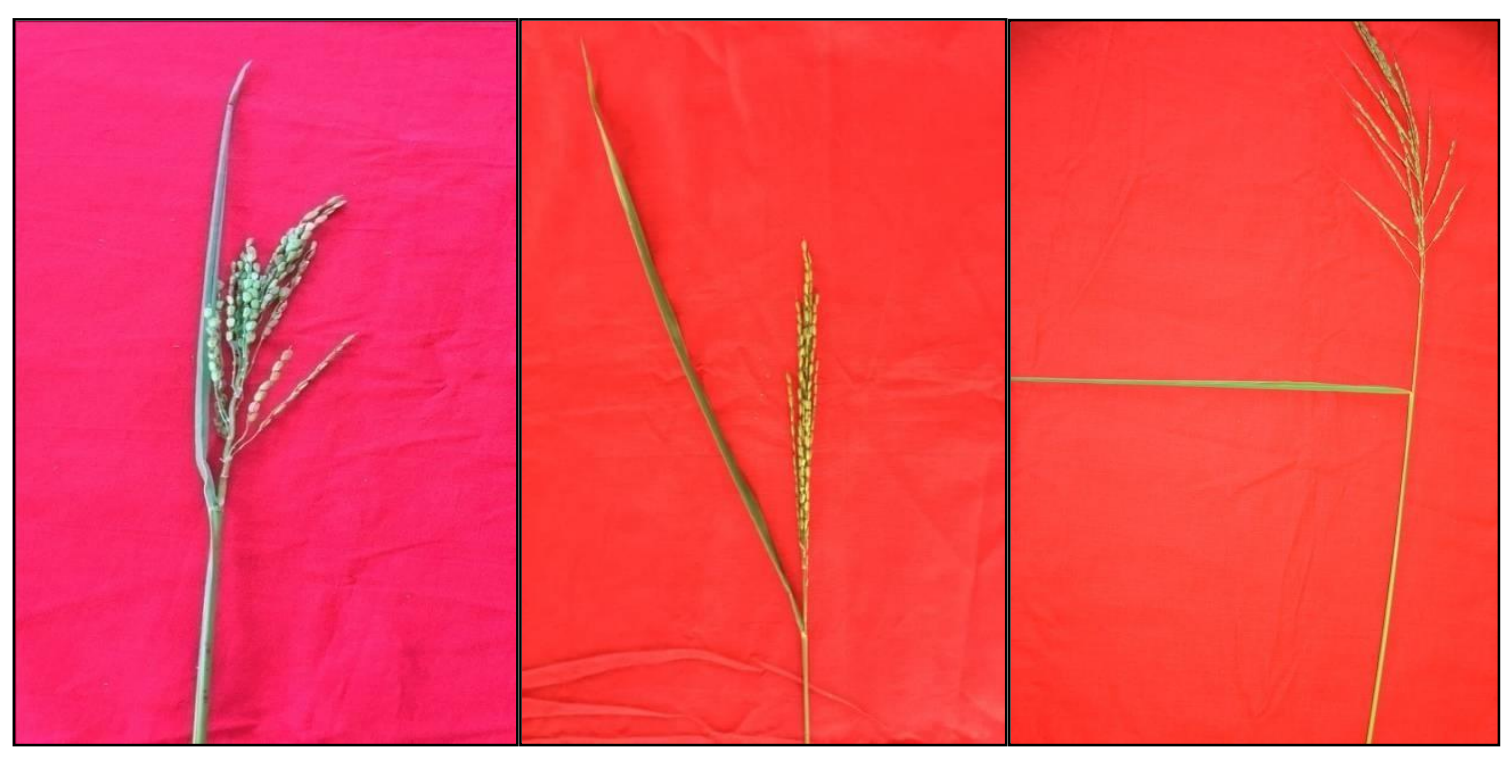

\begin{tabular}{|l|l|}
\hline Erect & Semi Erect \\
\hline
\end{tabular}

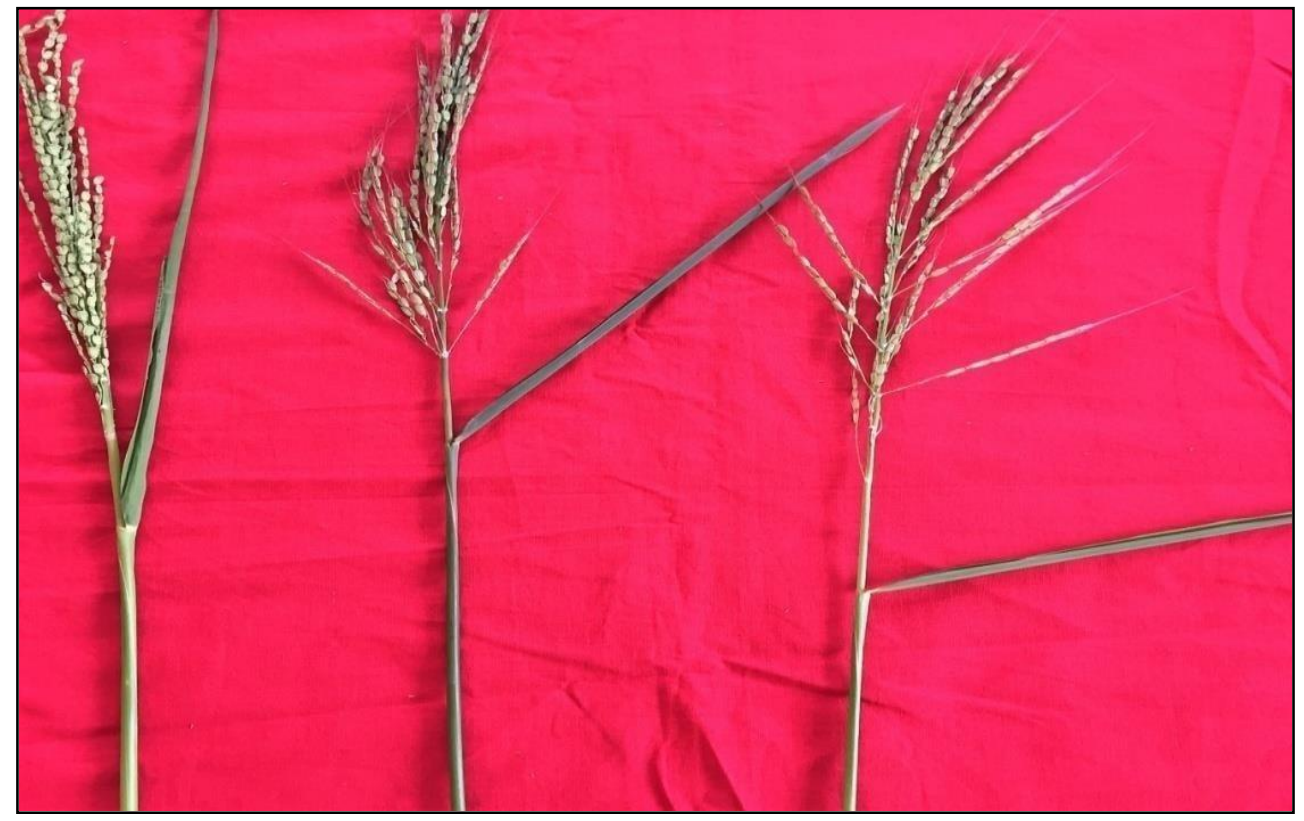


Plate.6a Variation in panicle awn

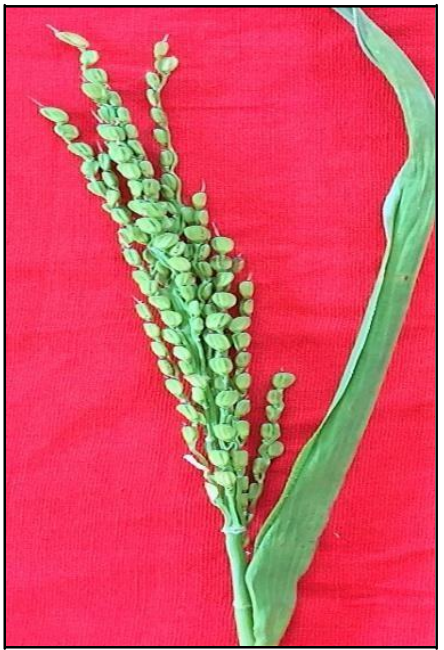

Short awned

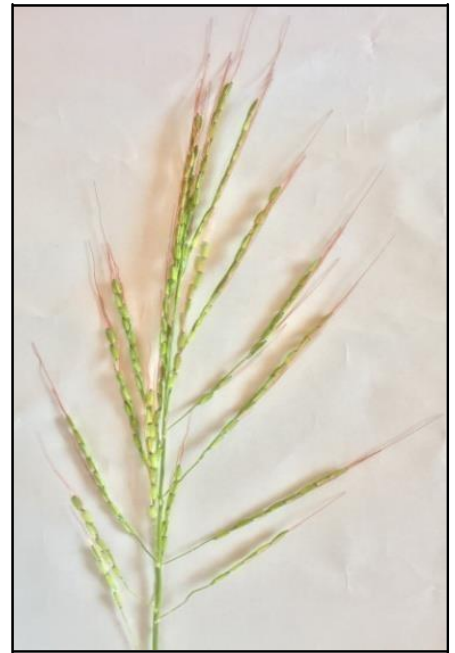

Long awned

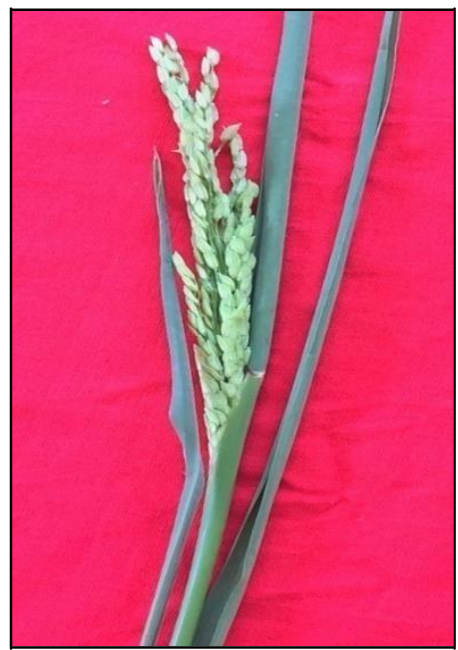

Awnless

Plate.6b Variation in stem anthocyanin colouration

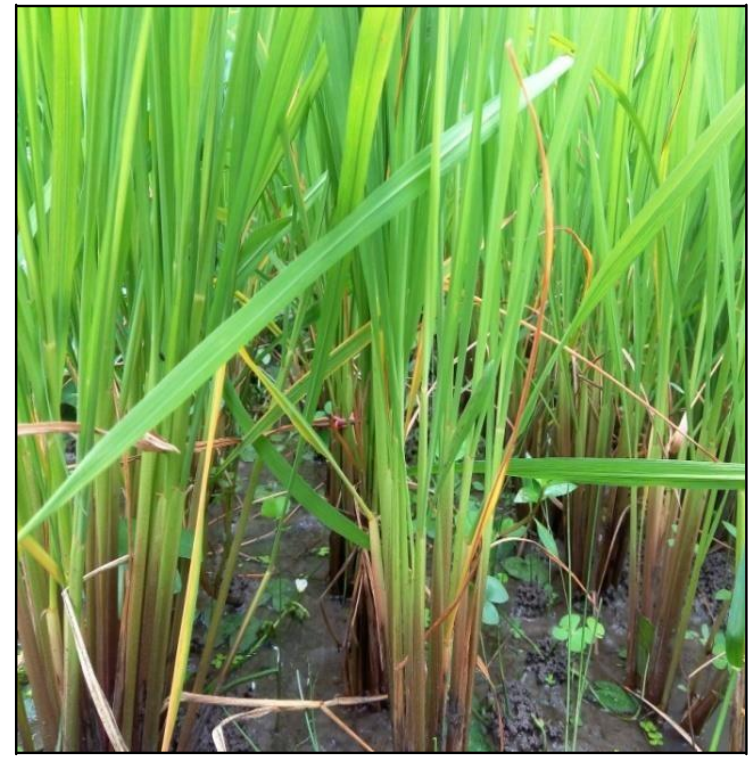

Anthocyanin pigmentation on stem

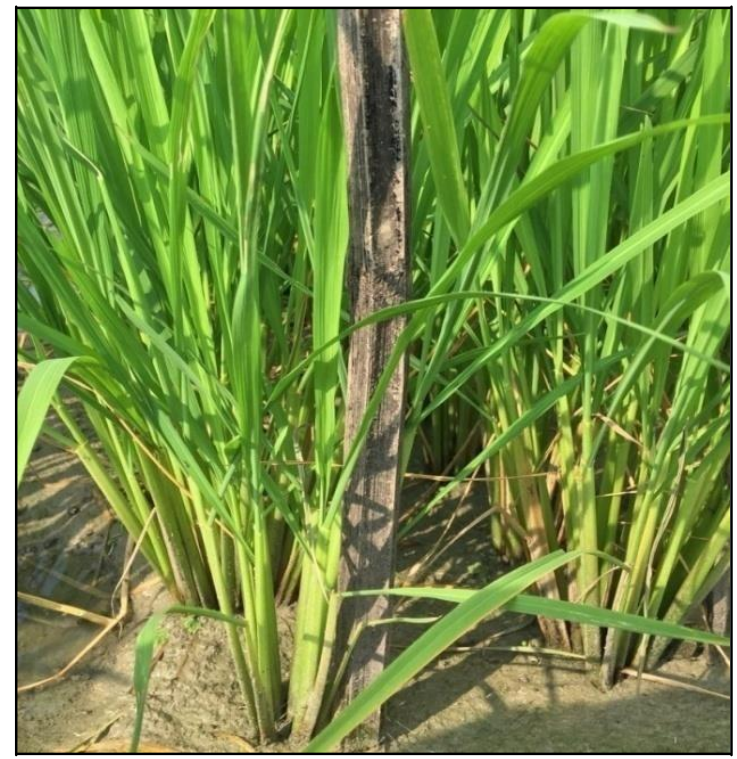

Absence of Anthocyanin on stem 
Plate.7 Variation in leaf intensity of green colour

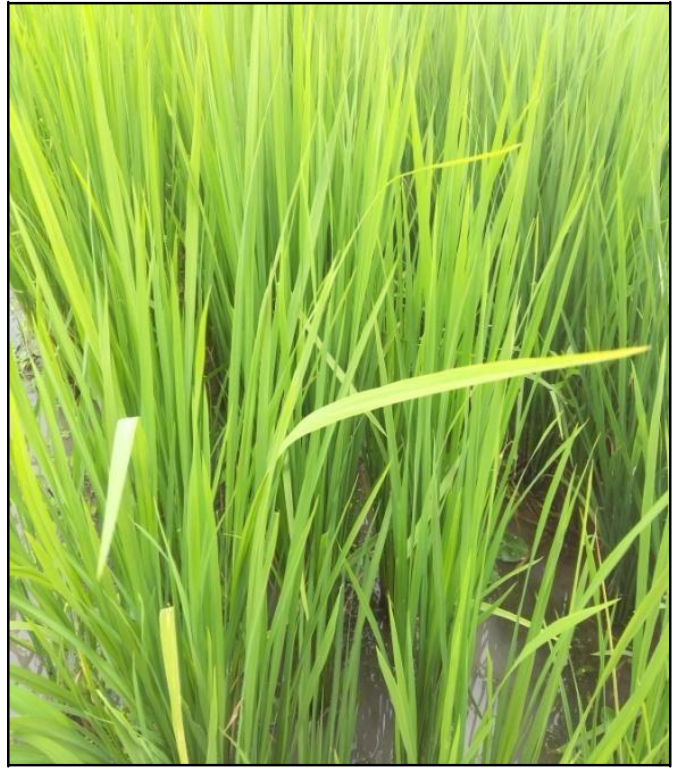

Light Green

The high estimates of heritability coupled with low genetic advance for no.of productive tillers per plant, grain yield per plant, and kernel length indicated the presence of non-additive gene effects. In the present study, three superior accessions, viz., EC861785, EC861804 and EC861813were found to be potential enough to be used as parents in various breeding programmes. These accessions recorded highest values for panicle length, productive tillers and grain yield per plant and hence their utilization in combination breeding may help in generating high yielding varieties by pyramiding all the favourable genes and keeping in view of the facts, much attention needs to be given for the components with high GCV, PCV and high heritability coupled with high genetic advance during selection for the further improvement of the remaining accessions.

In conclusion, among the eight morphological traits studied in the 31 accessions of $O$. glaberrima, three characters viz., absence of coleoptile colour, absence of leaf anthocyanin colouration and presence of leaf ligule were reported in all the 31 accessions. Remaining

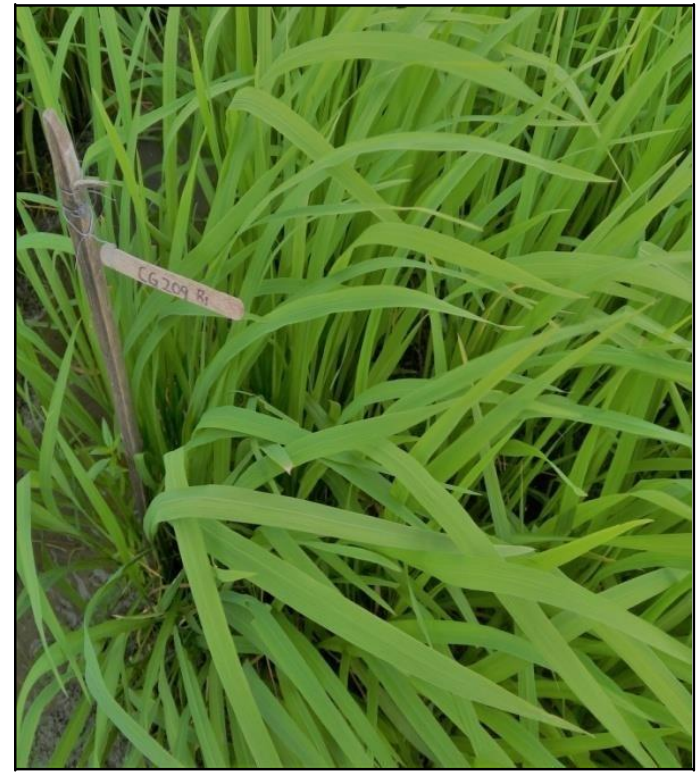

\section{Medium Green}

five characters were unique and distinct among the accessions. Out of 31 accessions of $O$. glaberrima, two accessions viz., EC 861804, EC 861805 were found to be highly distinct as they possessed distinct traits namely purple split ligule, medium green leaves, erect flag leaf, presence of awn along with lodging tolerance. Adequate genetic variability in the 31 accessions of $O$. glaberrima was observed.

The magnitude of PCV and GCV was high for number of productive tillers per plant, while high heritability coupled with high genetic advance was observed for days to 50 per cent flowering and number of spikelets per panicle. The accessions EC861785, EC861804 and EC861813 can be used as parental material in future rice breeding programme as they have recorded highest values for the important yield traits $v i z$, for panicle length, productive tillers and grain yield per plant. The present study revealed sufficient genetic variability for yield related traits which could be exploited for genetic improvement of rice cultivars (Oryza sativa $\mathrm{L}$ ). 


\section{References}

Abebe, T., Alamerew, S and Tulu, L. 2017. Genetic variability, heritability and genetic advance for yield and its related traits in rainfed lowland rice (Oryza sativa L.) genotypes at Fogera and Pawe, Ethiopia. Advanced Crop Science and Technology.5: 272.

Ahmad.,Qayyum., Sahibzada., Saleem., Ghaffar., Mehnaz and Farhad. 2011. Genetic diversity analysis for yield and other parameters in maize (Zea mays L.) genotypes. Asian Journal of Agricultural Sciences. 3.

Burton, G.W and DeVane, E.H. 1952. Estimating heritability in tall fescue (Festuca arundinacea) from replicated clonal material. Agronomy Journal. 19: 45.

Edukondalu, B., Ram Reddy, V., Shobha Rani, T., ArunaKumari, Ch and Soundharya, B. 2017. Studies on variability, heritability, correlation and path analysis for yield, yield attributes in rice (Oryza sativa L.).International Journal of Current Microbiology and Applied Sciences.6 (10): 2369-2376.

Fonfana, B and Cloutier, S. 2008. Assesment of molecular diversity with QTLs for preharvest sprouting resistance in wheat using microsatellite markers. Genome. 51: 375-386.

Indiastat.Agriculture Production Statistical Database.2016-17. http://www.indiastat. com.

Johnson, H.W., Robinson, H.F and Comstock, R.E. 1955. Estimation of genetic and environmental variability in soybean. Agronomy Journal. 47: 314-318.
Najeeb, S., Rather, A.G., Parray, G.A., Sheikh, F.A and Razvi, S.M. 2009.Studies on genetic variability, genotypic correlation and path coefficient analysis in maize under high altitude temperate ecology of Kashmir. Maize Genetics Cooperation Newsletter. 83: 1- 8.

Panse, V.G and Sukhatme, P.V. 1985. Statistical methods for Agriculture workers. Indian council of Agricultural research Publication. 87-89.

Raut, V.M. 2003. Qualitative genetics of Soyabean. Soybean Research. 1:1-28.

Rout B., Sridhar M., Muralidhara, B., Kamal Nath Reddy, KR., Sundaram R.M., Anantha M.S., Senguttuvel, P., SubbaRao, L.V, Padmavathi, G., Ranganath, H.K., Fiyaz, A.R., Jyothi, B., Suvarna Rani, C., Kalyani, M.B., Bidyasagar Mandal and Gireesh, C. 2017. Characterization of genetic diversity among wild rice accessions using genome specific In-Del markers. Journal of Rice Research.10 (2): 11-17.

Rusdiansyah, M., Subiono, T., Sunaryo, W., Suryadi, A., Sulastri, G and Anjasmara, S. 2017.The genetic diversity and agronomical characters of local cultivars of tidal rice in East Kalimantan, Indonesia. Biodiversitas. 18:1289-1293.

Sarla, N and Swamy, B.P.M. 2005.Oryza glaberrima: A source for improving Oryza sativa. Current science. 89 (6): 955-963.

Waqar, M.F., Malik., Rashid, M., Munir, M and Akram, Z. 2008. Evaluation and estimation of heritability and genetic advancement for yield related attributes in wheat lines. Pakistan Journal of Botany. 40 (4):1699-1702.

\section{How to cite this article:}

Ishwarya Lakshmi, V.G., C. Gireesh, M. Sreedhar, S. Vanisri, P.S. Basavaraj, B. Muralidhara, M.S. Anantha, G. Padmavathi, A.R. Fiyaz, B. Jyothi, C. Suvarna Rani, Bidyasagar Mandal and Subba Rao, L.V. 2018. Characterization of African Rice Germplasm for Morphological and Yield Attributing Traits. Int.J.Curr.Microbiol.App.Sci. 7(12): 1288-1303.

doi: https://doi.org/10.20546/ijcmas.2018.712.159 